\title{
Masses for the Local Group and the Milky Way
}

\author{
Yang-Shyang $\mathrm{Li}^{1 \star}$ and Simon D. M. White ${ }^{2} \dagger$ \\ ${ }^{1}$ Kapteyn Astronomical Institute, PO Box 800, 9700 AV, Groningen, The Netherlands \\ ${ }^{2}$ Max-Planck-Institut für Astrophysik, Karl-Schwarzschild-Str. 1, D-85748 Garching, Germany
}

Accepted. Received ; in original form

\begin{abstract}
We use the very large Millennium Simulation of the concordance $\Lambda$ CDM cosmogony to calibrate the bias and error distribution of Timing Argument estimators of the masses of the Local Group and of the Milky Way. From a large number of isolated spiral-spiral pairs similar to the Milky Way/Andromeda system, we find the interquartile range of the ratio of timing mass to true mass to be a factor of 1.8 , while the $5 \%$ and $95 \%$ points of the distribution of this ratio are separated by a factor of 5.7. Here we define true mass as the sum of the "virial" masses $M_{200}$ of the two dominant galaxies. For current best values of the distance and approach velocity of Andromeda this leads to a median likelihood estimate of the true mass of the Local Group of $5.27 \times 10^{12} \mathrm{M}_{\odot}$, or $\log M_{L G} / M_{\odot}=12.72$, with an interquartile range of $[12.58,12.83]$ and a $5 \%$ to $95 \%$ range of $[12.26,13.01]$. Thus a $95 \%$ lower confidence limit on the true mass of the Local Group is $1.81 \times 10^{12} \mathrm{M}_{\odot}$. A timing estimate of the Milky Way's mass based on the large recession velocity observed for the distant satellite Leo I works equally well, although with larger systematic uncertainties. It gives an estimated virial mass for the Milky Way of $2.43 \times 10^{12} \mathrm{M}_{\odot}$ with a $95 \%$ lower confidence limit of $0.80 \times 10^{12} \mathrm{M}_{\odot}$.
\end{abstract}

Key words: Galaxy: formation - galaxies: Local Group - galaxies: kinematics and dynamics - dark matter.

\section{INTRODUCTION}

During the 1970's it became generally accepted that most, per' haps all, galaxies are surrounded by extended distributions of dark matter, so-called dark halos (Einasto, Kaasik \& Saar 1974; Ostriker, Peebles \& Yahil 1974). These were soon understood to play an essential role in driving the formation and clustering of galaxies (White \& Rees 1978). With the introduction of the Cold Dark Matter (CDM) paradigm, these ideas took more concrete form, allowing quantitative predictions to be made both for the population properties (Blumenthal et al. 1984) and for the large-scale clustering (Davis et al. 1985) of galaxies.

Measurements of the fluctuation spectrum of the Cosmic Microwave Background (Smoot et al. 1992; Spergel et al. 2003) and of the apparent acceleration of the cosmic expansion (Riess et al. 1998; Perlmutter et al. 1999) elevated the CDM model, in its variant with a cosmological constant $(\Lambda \mathrm{CDM})$, to the status of a standard paradigm. At the same time improving numerical techniques and faster computers have enabled detailed simulation of the formation and evolution of the galaxy population within this paradigm throughout a significant fraction of the observable Universe (Springel et al. 2005). Nevertheless, direct observational evidence for halos as extended as the paradigm predicts around galaxies like our own has so far come only from statistical analyses of the

\footnotetext{
* Email: ysleigh@astro.rug.nl

† Email: swhite@MPA-Garching.MPG.DE
}

dynamics of satellite galaxies (e.g. Zaritsky et al. 1997; Prada et al. 2003) and of the gravitational lensing of background galaxies (e.g. Seljak 2002; Mandelbaum et al. 2006) based on large samples of field spirals.

The earliest observational indication that the effective mass of the Milky Way must be much larger than its stellar mass came from the Timing Argument (hereafter TA) of Kahn \& Woltier (1959). These authors noted that the Local Group is dominated by the two big spirals, and that these are currently approaching each other at about $100 \mathrm{~km} \mathrm{~s}^{-1}$. (The next most luminous galaxy is M33 which is probably about a factor of 10 less massive than M31 and the Galaxy.) This reversal of the overall cosmic expansion must have been generated by gravitational forces, and since the distance to the nearest external bright galaxy is much greater than that between M31 and the Milky Way, these forces are presumably dominated by material associated with the two spirals themselves.

Kahn \& Woltjer set up a simple model to analyse this situation - two point masses on a radial orbit. These were at pericentre (i.e. at zero separation) at the Big Bang and must have passed through apocentre at least once in order to be approaching today. Clearly this requires an apocentric separation larger than the current separation and an orbital period less than twice the current age of the Universe. Together these requirements put a lower limit on on the total mass of the pair. A more precise estimate of the minimum possible mass is obtained from the parametric form of Kepler's laws for a zero angular momentum orbit: 
$r=a(1-\cos \chi)$

$t=\left(\frac{a^{3}}{G M}\right)^{1 / 2}(\chi-\sin \chi)$

$\frac{d r}{d t}=\sqrt{\frac{G M}{a}} \frac{\sin \chi}{1-\cos \chi}$

where $r$ is the current separation, $d r / d t$ is the current relative velocity, $a$ is the semi-major axis, $\chi$ is the eccentric anomaly, $t$ is the time since the Big Bang (the age of the universe) and $M$ is total mass (Lynden-Bell 1981). Given observationally determined values for $r, d r / d t$ and $t$, these equations have an infinite set of discrete solutions for $\chi, a$ and $M$ labelled by the number of apocentric passages since the Big Bang. The solution corresponding to a single apocentric passage gives the smallest (and only plausible) estimate for the mass, which is about $5 \times 10^{12} \mathrm{M}_{\odot}$ for current estimates of $r, d r / d t$ and $t$. Note that this is still a lower limit on the total mass, even within the simple point-mass binary model, since any non-radial motions in the system would increase its present kinetic energy and so increase the mass required to reverse the initial expansion and bring the pair to their observed separation by the present day (see Einasto \& Lynden-Bell 1982).

As Kahn \& Woltier realised, this timing estimate of the total mass of the Local group exceeds by more than an order of magnitude the mass within the visible regions of the galaxies, as estimated from their internal dynamics, in particular, from their rotation curves. Thus $90 \%$ of the mass must lie outside the visible galaxies and be associated with little or no detectable light. Modern structure formation theories like $\Lambda \mathrm{CDM}$ predict this mass to be in extended dark matter halos with $M(r)$ increasing very roughly as $r$ out to the point where the halos of the two galaxies meet. Such structures have no well-defined edge, so any definition of their total mass is necessarily somewhat arbitrary. In addition, their dynamical evolution from the Big Bang until the present is substantially more complex than that of a point-mass binary. Thus the mass value returned by the Timing Argument cannot be interpreted without some calibration against consistent dynamical models with extended dark halos.

A first calibration of this type was carried out by Kroeker \& Carlberg (1991) using simulations of an Einstein-de Sitter CDM cosmogony. Here we use the very much larger Millennium Simulation (Springel et al. 2005) to obtain a more refined calibration based on a large ensemble of galaxy pairs with observable properties similar to those of the Local Group. We find that the standard timing estimate is, in fact, an almost unbiased estimate of the sum of the conventionally defined virial masses of the two large galaxies.

Zaritsky et al. (1989) attempted to measure the halo mass of the Milky Way alone by measuring radial velocities for its dwarf satellites and assuming the population to be in dynamical equilibrium in the halo potential. They noted, however, that one of the most distant dwarfs, Leo I, has a very large recession velocity and as a result provides a interesting lower limit on the Milky Way's mass by a variant of the original Timing Argument. To reach its present position and radial velocity, Leo I must have passed pericentre at least once since the Big Bang and now be receding from the Galaxy for (at least) the second time.

Applying the point-mass radial orbit Equations (1) - (3) to this case gives a lower bound of about $1.6 \times 10^{12} \mathrm{M}_{\odot}$. This seems likely to be a significant underestimate, since Leo I could not have passed through the centre of the Milky Way without being tidally destroyed so its orbit cannot be purely radial. Below we calibrate the Timing Argument for this case also, finding it to work well although with significantly more scatter than for the Local Group as a whole. This is because the $\Lambda \mathrm{CDM}$ paradigm predicts that the dynamics on the scale of Leo I's orbit ( $\sim 200 \mathrm{kpc})$ is typically more complex than on the scale of the Local Group as a whole $(\sim 700 \mathrm{kpc})$.

Our paper is organised as follows. In Section 2 we briefly describe the Millennium Simulation and the selection criteria we use to define various samples of 'Local Group-like' pairs and of 'Milky Way-like' halos. In Section 3 we plot the ratio of true total mass to Timing Argument mass estimate for these samples, and we use its distribution to define an unbiased TA estimator of true mass with its associated confidence ranges. In Section 3.2 this is then applied to the Local Group in order to obtain an estimate its true mass with realistic uncertainties. Section 3.3 attempts to carry out a similar calibration for the TA-based estimate of the Galaxy's halo mass from the orbit of Leo I. We conclude in Section 4 with a summary and brief discussion of our results.

\section{THE MILLENNIUM SIMULATION}

The Millennium Simulation is an extremely large cosmological simulation carried out by the Virgo Consortium (Springel et al. 2005). It followed the motion of $N=2160^{3}$ dark matter particles of mass $8.6 \times 10^{8} h^{-1} \mathrm{M}_{\odot}$ within a cubic box of comoving size $500 h^{-1} \mathrm{Mpc}$. Its comoving spatial resolution (set by the gravitational softening) is $5 h^{-1} \mathrm{kpc}$. The simulation adopted the concordance $\Lambda \mathrm{CDM}$ model with parameters $\Omega_{m}=$ $0.25, \Omega_{b}=0.045, h=0.73, \Omega_{\Lambda}=0.75, n=1$ and $\sigma_{8}=$ 0.9 , where, as usual, we define the Hubble constant by $H_{0}=$ $100 \mathrm{~km} \mathrm{~s}^{-1} \mathrm{Mpc}^{-1}$. The current age of the universe is then $13.6 \times 10^{9} \mathrm{yr}$. The positions and velocities of all particles were stored at 63 epochs spaced approximately logarithmically in expansion factor at early times and at approximately $300 \mathrm{Myr}$ intervals after $z=2$. For each such snapshot a friends-of-friends group-finder was used to locate all virialised structures, and their self-bound substructures (subhalos) were identified using SUBFIND (Springel et al. 2001). Halos and subhalos in neighbouring outputs were then linked in order to build formation history trees for all the subhalos present at each time. These data are publicly available at the Millennium release site1. A "Milky Way" halo at $z=0$ typically contains a few thousand particles and several resolved subhalos.

Galaxy formation was simulated within these merging history trees by using semi-analytic models to follow the evolution of the baryonic components associated with each halo/subhalo. Processes included are radiative cooling of diffuse gas, star formation, the growth of supermassive black holes, feedback of energy and heavy elements from supernovae and AGN, stellar population evolution, galaxy merging and effects due to a reionising UV background. The $z=0$ galaxy catalogue we analyse here corresponds to the specific model of Croton et al. (2006) and details of its assumptions and parameters can be found in that paper. Data for the galaxy population at all redshifts are available at the Millennium web site for the updated model of De Lucia \& Blaizot (2007), as well as for the independent galaxy formation model of Bower et al. (2006). All these models are tuned to fit a wide variety of data on the nearby galaxy population, and in addition fit many (but not all!) available data at 
Masses for the Local Group and the Milky Way

higher redshift (see, for example, Kitzbichler \& White 2006). The details of the galaxy formation modelling are not, however, important for the dynamical issues which are the focus of our own paper.

At $z=0$ there are $18.2 \times 10^{6}$ halos/subhalos identified in the simulation to its resolution limit of 20 particles. The galaxy formation model populates these with $8,394,180$ galaxies brighter than an absolute magnitude limit of $M_{B}=-16.7$ above which the catalogue can be considered reasonably complete. These catalogues list a number of properties for the halos, subhalos and galaxies which will be important for us. Galaxies are categorised into three types according to the nature of their association with the dark matter. A Type 0 galaxy sits at the centre of the dominant or main subhalo and can be considered the central galaxy of the halo itself (formally, of the FOF group). A Type 1 galaxy sits at the centre of one of the smaller non-dominant subhalos associated with a FOF group. Finally, a Type 2 galaxy is associated to a specific particle and no longer has an associated subhalo because the object within which it formed was tidally disrupted after accretion onto a larger halo. Such galaxies merge with the central galaxy of their new halo after waiting for a dynamical friction time.

Each galaxy in the catalogue has an associated "rotation velocity" $V_{\max }$. This is the maximum of the circular velocity $V_{c}(r)=$ $(G M(r) / r)^{1 / 2}$ of its subhalo for Types 0 and 1; for Type 2 objects $V_{\max }$ is frozen to its value at the latest time when the galaxy still occupied a subhalo. Type 0 and 1 galaxies also have an associated mass $M_{\text {halo }}$ which is the mass of the self-bound subhalo which surrounds them. Finally, halos of Type 0 galaxies have a conventional "virial mass" $M_{200}$, defined as the total mass within the largest sphere surrounding them with an enclosed mean density exceeding 200 times the critical value. Below we will consider both $M_{\text {halo }}$ and $M_{200}$ as possible definitions for the "true" masses of M31 and the Galaxy.

We use the Millennium Simulation to construct samples of mock Milky Way/Andromeda galaxies and of mock Local Groups as follows. We begin by identifying all Type 0 or Type 1 galaxies with characteristic "rotation velocity" either in the narrow range $200 \leqslant V_{\max }<250 \mathrm{kms}^{-1}$ or in the wider range, $150 \leqslant$ $V_{\max }<300 \mathrm{kms}^{-1}$. This produces samples of 166,090 and 699, 177 galaxies respectively. The exclusion of Type 2 galaxies reduces the samples by about 5-6\% in each case, but the excluded galaxies are in any case not plausible analogues for the Local Group giants since they are almost all members of large groups or clusters. We also consider subsamples in which the morphologies predicted by the semianalytic model are forced to approximate those of M31 and the Galaxy. Specifically, we require a bulge-to-total luminosity ratio in the range $1.2 \leqslant M_{B \text {,bulge }}-M_{B \text {,total }}<2.5$ so that the disks are 2 to 9 times brighter than the bulges in the $B$-band. This morphology cut reduces the samples in the two $V_{\max }$ ranges to 62,605 and 271,857 galaxies respectively.

We then identify Local Group analogues in each of these four samples by identifying isolated pairs with separations in the range of $500-1,000 \mathrm{kpc}$ and with negative relative radial velocities. (Note that this is the true relative velocity rather than the relative peculiar velocity, i.e. we have added the Hubble expansion to the relative peculiar velocity and have required the result to be negative.) We identify isolated pairs by keeping only those which have no "massive" companion, defined as a galaxy with $V_{\text {max }} \geqslant 150 \mathrm{~km} \mathrm{~s}^{-1}$, within a sphere of $1 \mathrm{Mpc}$ radius centred on the mid-point of the binary, and no nearby cluster, defined as a halo with $M_{200}>3 \times 10^{13} \mathrm{M}_{\odot}$ within $3 \mathrm{Mpc}$ of the mid-point of the binary. These cuts ensure that the dynamics are dominated by mass associated with the two main systems, as appears to be the case for the Local Group. For galaxies selected in the narrower $V_{\max }$ range we then find 178 pairs when the morphology cut is applied and 1, 128 pairs when it is not. For the wider $V_{\max }$ range the corresponding numbers are 2, 815 pairs and 16,479 pairs respectively.

When calibrating the TA estimator it proves advantageous to use simulated pairs with dynamical state quite close to that of the real Local Group. As we will see below, this eliminates some systems where the dominant motion is not in the radial direction and the TA therefore significantly underestimates the mass. We therefore make one final cut which requires the approach velocity of the two galaxies to lie between 0.5 and 1.5 times the value measured for the real system $\left(-130 \mathrm{~km} \mathrm{~s}^{-1}\right)$. This results in our final sets of Local Group lookalikes. For the narrower $V_{\max }$ range we end up with 117 pairs when the morphology cut is applied and 758 pairs when it is not, while for the wider $V_{\max }$ range the corresponding numbers are 1,273 pairs and 8,449 pairs respectively.

When we study the application of the Timing Argument to the Milky Way-Leo I system, we consider individual galaxies from both our $V_{\max }$ ranges. We require these to be isolated by insisting that there should be no bright/massive companion (with luminosity exceeding $10 \%$ of that of the host or $V_{\max }>150 \mathrm{~km} \mathrm{~s}^{-1}$ ) closer than $700 \mathrm{kpc}$ and no massive group (defined as above) closer than $3 \mathrm{Mpc}$. This produces samples of 137, 926 and 266, 229 potential hosts in the cases with and without the morphology cut for the wider $V_{\max }$ range, and 29, 245 and 57, 816 potential hosts for the narrower range. We then search for Leo I analogues around these hosts by identifying companions in the separation range 200 to $300 \mathrm{kpc}$ with $V_{\max }(\mathrm{comp}) \leqslant 80 \mathrm{~km} \mathrm{~s}^{-1}, M_{B}<-16.7$ and $V_{r a} \geqslant 0.7 V_{\max }$ (host) where $V_{r a}$ is the relative radial velocity of the two objects and the last condition reflects the fact that Leo I is useful for estimating the Milky Way's mass only because its recession velocity is comparable to the Galactic rotation velocity $\left(V_{r a} \sim 0.8 V_{\max }(\right.$ host) for the real Leo I-Milky Way system). Pairs sharing the same MW-like host are excluded in the final list.

With these cuts we find 344 and 896 satellite-host pairs in the samples with and without the morphology cut for the looser $V_{\max }$ range, and 168 and 374 for the tighter range. These relatively small numbers reflect the fact that only about $10 \%$ of potential hosts actually have a faint companion in this distance range which is still bright enough to be resolved, and fewer than $5 \%$ of these satellites are predicted to have positive recession velocities comparable to that observed.

\section{RESULTS}

\subsection{Calibration of the Timing Argument mass for the Local Group}

For each simulated Local Group analogue the separation and relative radial velocity of the two galaxies can be combined with the age of the Universe (taken to be $13.6 \mathrm{Gyr}$ ) to obtain a Timing Argument mass estimate $M_{T A}$ (Equations (1) to (3)). The true mass of the pair $M_{t r}$ is harder to define because of the extended and complex mass distributions predicted by the $\Lambda$ CDM model. The mass of an individual dark halo is often taken to be $M_{200}$ the mass within a sphere of mean density 200 times the critical value, so a natural choice for $M_{t r}$ is the sum of $M_{200}$ for the two galaxies. The Millennium Simulation database only lists $M_{200}$ for Type 0 galaxies, those at the centre of the main subhalo of each friends-of-friends particle group. Many of our LG analogues lie within a single FOF group. One of the pair is then a Type 1 galaxy, the central object 
of a subdominant subhalo, and so has no listed value for $M_{200}$. In such cases we have gone back to the particle data for the simulation in order to measure $M_{200}$ directly also for these galaxies.

An alternative convention is to define $M_{t r}$ as the sum of the values of $M_{\text {halo }}$, the maximal self-bound mass of each subhalo; this is included in the database for both Type 0 and Type 1 galaxies. In the following we use the notation $M_{t r, 200}$ and $M_{t r, \text { halo }}$ to distinguish these two definitions. For either we can calculate the ratio of true mass to Timing Argument estimate,

$A_{x}=M_{t r, x} / M_{T A}$,

where the suffix $x$ is 200 or halo depending on the definition adopted for $M_{t r}$. If the Timing Argument is a good estimator of true mass, our samples of LG analogues should produce a narrow distribution of $A$ values. This distribution then allows the TA mass estimate for the real Local Group to be converted into a best estimate of its true mass, together with associated confidence intervals.

Our preferred sets of Local Group analogues contain simulated galaxy pairs which mimic the real system in terms of morphology, isolation, pair separation and pair approach velocity. In addition, they require the halos of the simulated galaxies to have $V_{\max }$ values within about $\pm 10 \%$ and $\pm 35 \%$ of those estimated for M31 and the Galaxy for the tight and loose ranges of $V_{\max }$, respectively. In order to understand the influence of these constraints we give results below not only for our "best" samples but also for samples where the various constraints are relaxed. Thus, we consider samples in which 1) both morphology and isolation requirements are applied (our preferred case), 2) the isolation requirement is removed, 3) the morphology requirement is removed, and 4) both morphology and isolation requirements are removed. For each case, we compare results for the two allowed ranges of $V_{\max }$ and we also examine the effect of loosening the radial velocity constraint to $V_{r a}<0$.

Fig. 11 gives histograms of the distribution of $A_{200}$ for a sample in the narrow $V_{\max }$-range with our preferred isolation, morphology and radial velocity cuts, as well as for three samples with the same $V_{\max }$ and $V_{r a}$ cuts but with reduced morphology and isolation requirements. Fig. 2 presents these same distributions in cumulative form and compares them with the corresponding distributions for samples with the loosened circular velocity requirement, $150 \mathrm{~km} \mathrm{~s}^{-1} \leqslant V_{\max }<300 \mathrm{~km} \mathrm{~s}^{-1}$. In both plots black curves refer to class (1) samples for which both isolation and morphology cuts are imposed, while red, green and blue curves refer to samples in classes (2), (3) and (4) respectively. Results for the broader $V_{\max }$ selection are indicated by dashed curves in Fig. 2 We give numerical results for various percentile points of these distributions in Table 1 and repeat all these in Table 2 for samples where the separation velocity requirement has been loosened to $V_{r a}<0$.

The first and most important point to note from these these figures and tables is that the median value of $A_{200}$ is very robust and only varies between 0.98 and 1.34 for our full range of sample selection criteria. With our preferred cuts the median values are 1.15 and 0.99 for the narrow and wide $V_{\max }$ samples respectively. The best estimate of the true mass of the Local Group (for this definition) is thus very similar to its Timing Argument mass estimate, and depends very little on the calibrating sample of simulated pairs.

The second important point is that the width of the distribution of $A_{200}$ does depend on how the calibrating sample is defined. In particular, it is narrower for samples with the more restrictive $V_{\max }$ range, and for given $V_{\max }$ range it is smallest for samples with our preferred cuts, those which match the dynamical and morphological properties of the Local Group most closely. For the narrow

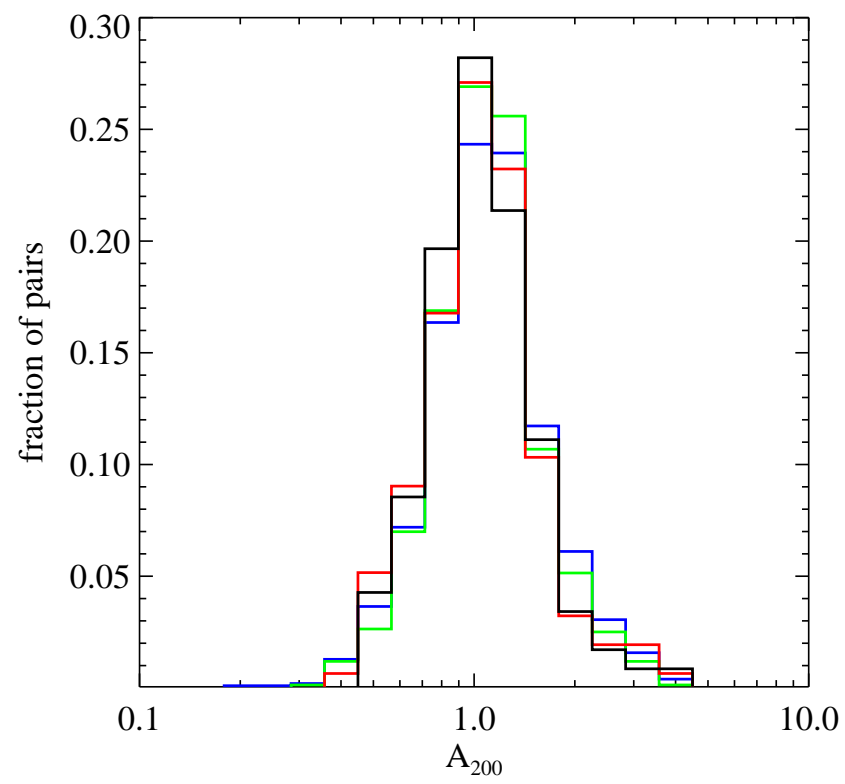

Figure 1. Normalised histograms of $A_{200}$, the ratio of true mass to Timing Argument estimate, for samples of Local Group analogues with $200 \mathrm{kms}^{-1} \leqslant V_{\max }<250 \mathrm{kms}^{-1}$ and $-195 \mathrm{kms}^{-1}<V_{\text {ra }}<$ $-65 \mathrm{~km} \mathrm{~s}^{-1}$. The black histogram refers to our preferred selection where both isolation and morphology requirements are imposed. For the red histogram the isolation requirement has been removed, for the green histogram the morphology requirement, and for the blue histogram both requirements.

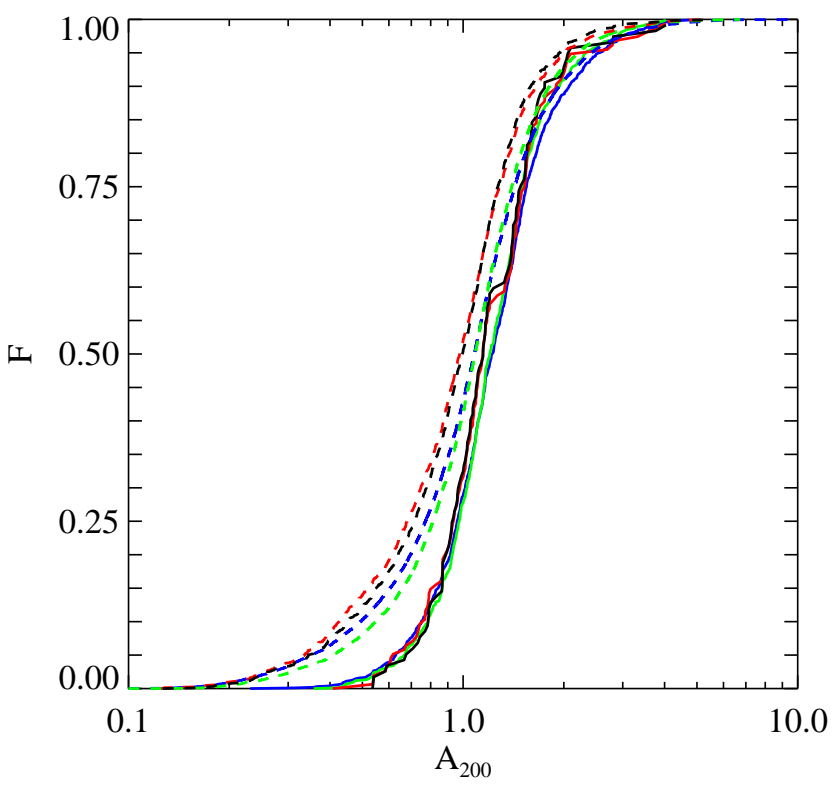

Figure 2. Cumulative distributions of $A_{200}$ the ratio of true mass to Timing Argument estimate for Local Group analogues with $-195 \mathrm{~km} \mathrm{~s}^{-1}<$ $V_{r a}<-65 \mathrm{~km} \mathrm{~s}^{-1}$. The solid curves correspond to the four samples already plotted in Fig. 1 while the dashed curves are for samples with $150 \mathrm{~km} \mathrm{~s}^{-1} \leqslant V_{\max }<300 \mathrm{~km} \mathrm{~s}^{-1}$. The colour coding is the same as in Fig.1 black indicates samples with our preferred isolation and morphology constraints. 
Masses for the Local Group and the Milky Way

$V_{\max }$ sample the interquartile range is a factor of just 1.6, and the upper and lower $5 \%$ points are separated by a factor of 3 . For the wider velocity range the interquartile range is a factor of 1.8 and the $5 \%$ points are separated by a factor of 5.7 . This shows the Timing Argument to be remarkably precise for systems similar to the Local Group.

The broadening of the distribution as the selection requirements are relaxed is easy to understand. Removing the isolation requirements allows third bodies to play a significant role in the dynamics. This can extend the upper tail of the $A_{200}$ distribution if mass from the third body falls inside $R_{200}$ for one of the pair galaxies or if the gravity of the third galaxy produces a tidal field which opposes the attraction between the pair members. It can extend the lower tail if the mass of the third body lies between the pair members but outside their $R_{200}$ spheres, thus enhancing their mutual attraction without adding to their mass. Removing the morphology constraint moves the whole distribution towards larger values and this effect is most pronounced in the large $A_{200}$ tail. This is because objects with more dominant bulges have more complex merger histories. They typically form in denser regions and their halos tend to be more massive and to have more complex structure.

Loosening the requirements on $V_{\max }$ affects the distribution in a complex way. There is a tight relation between $V_{\max }$ and $M_{200}$ (also $M_{\text {halo }}$ ) in the $\Lambda$ CDM structure formation model (e.g. Navarro, Frenk \& White 1997). Thus if we place tight restrictions on the $V_{\max }$ values of our galaxies, we will obtain a sample of Local Group analogues with a narrow range of $M_{t r}$ values. If, in addition, we force the parameters which enter in the Timing Argument (the pair separation and relative radial velocity) to lie in narrow ranges, then the TA mass estimate itself is tightly constrained. The distribution of $A_{200}$ is thus forced to be narrow as a consequence of our selection criteria.

A second effect is that most of the new pairs added by widening the requirement on $V_{\max }$ have at least one galaxy with $150 \mathrm{~km} \mathrm{~s}^{-1} \leqslant V_{\max }<200 \mathrm{~km} \mathrm{~s}^{-1}$, thus with relatively low $M_{t r}$. This simply reflects the strong dependence of halo abundance on $V_{\max }$. Given that halo mass scales approximately as $V_{\max }^{3}$ it is striking that the addition of so many pairs containing a "low mass" galaxy reduces the median value of $A_{200}$ by just $15 \%$. The low tail of the distribution is more strongly affected, by almost a factor of 2 at the lower 5\% point, but the upper end of the distribution is barely affected at all. This demonstrates that the main body of the distribution is weakly affected by restrictions on $V_{\max }$, but that the lower tail (which is needed to place a lower limit on the true mass of the Milky Way) is suppressed if $V_{\max }$ is not allowed to take small values.

In Table 2 we show the effect of weakening the cut on relative radial velocity to require only that the two main galaxies be approaching. Again this has remarkably little effect on the median $A_{200}$ values. A comparison with Table 1 shows them all to be increased by about $10 \%-15 \%$. The effects on the tails of the distributions are more substantial. The $95 \%$ point is typically increased by about a factor of 2 . This is because the sample now includes a substantial number of pairs with small $V_{r a}$ (and thus smaller TA mass estimate) for which tangential motion is important for their current orbit. The 5\% point of the distribution is significantly reduced, reflecting the fact that our restrictions on relative approach velocity exclude a non negligible number of systems with approach velocities larger than $195 \mathrm{~km} \mathrm{~s}^{-1}$, and thus with large TA mass estimates. Such systems must have more mass outside the conventional virial radii of the two galaxies than do typical Local Group analogues in our samples.
In conclusion, we believe our most precise and robust estimate of the distribution of $A_{200}$ to be that obtained with our preferred morphology, isolation and radial velocity cuts for $150 \mathrm{~km} \mathrm{~s}^{-1} \leqslant$ $V_{\max }<300 \mathrm{~km} \mathrm{~s}^{-1}$, and we will use this distribution in the next section to estimate the true mass of the Local Group. Although the tails of the distribution are suppressed still further for a narrower range of $V_{\max }$, this is at least in part due to the artificial effects mentioned above. In addition the number of Local Group analogues is too small in this case for the tails of the distribution to be reliably determined. From Table 1 we see that the best estimate of the true mass of the Local Group (which we take to be that obtained using the median value of $A_{200}$ ) is almost identical to the direct TA estimate. The most probable range of true mass (given by the quartiles of $A_{200}$ ) extends to values about $30 \%$ above and below this, while the $95 \%$ confidence lower limit on the true mass (given by the $5 \%$ point of the $A_{200}$ distribution) is a factor of 2.9 smaller.

\subsection{Application to the Local Group}

The three observational parameters needed to make a Timing Argument mass estimate for the Local Group are the separation between the two main galaxies, their radial velocity of approach and the age of the Universe. The latter is now determined to high precision through measurements of microwave background fluctuations. Spergel et al. (2007) give $13.73 \pm 0.16 \mathrm{Gyr}$. The distance to M31 is also known to high precision. We adopt the value $784 \pm 21 \mathrm{kpc}$ given by Stanek \& Garnavich (1998) based on red clump stars, noting that it agrees almost exactly with the slightly less precise value obtained by Holland (1998) from fits to the colourmagnitude diagrams of M31 globular clusters. Although the heliocentric recession velocity of M31 is known even more precisely $\left(-301 \pm 1 \mathrm{~km} \mathrm{~s}^{-1}\right.$ according to Courteau \& van den Bergh 1999) the approach velocity of the two giant galaxies is less certain because of the relatively poorly known rotation velocity of the Milky Way at the Solar radius. van der Marel \& Guhathakurta (2007) go through a careful analysis of the uncertainties and conclude that $V_{r a}=130 \pm 8 \mathrm{~km} \mathrm{~s}^{-1}$. Inserting these modern values into Equations (1) to (3) we obtain our Timing Argument estimate of the mass of the Local Group:

$M_{L G, T A} \approx 5.32 \pm 0.48 \times 10^{12} \mathrm{M}_{\odot}$,

where the uncertainty is dominated by that in the relative radial velocity. This uncertainty is still small in comparison to the scatter in the ratio of true mass to TA estimate, so we will neglect it in the following. The apocentric distance of the implied relative orbit of the two galaxies is $1103 \pm 30 \mathrm{kpc}$.

We now combine this Timing Argument estimate with the distribution of $A_{200}$ obtained in the last section for our most precise and reliable sample of Local Group analogues (the sample with our preferred morphology, isolation and radial velocity cuts and with the wider allowed range of $V_{\max }$ ) to obtain our best estimate of the true mass of the Local Group, defined here as the sum of the $M_{200}$ values of the two main galaxies:

$M_{L G, \text { true }}=5.27 \times 10^{12} \mathrm{M}_{\odot}$,

or $\log M_{L G \text {,true }} / M_{\odot}=12.72$. The most plausible range for this quantity is then $[12.58,12.83]$ with a $95 \%$ confidence lower limit of 12.26 , i.e $M_{L G \text {,true }}>1.81 \times 10^{12} \mathrm{M}_{\odot}$ at $95 \%$ confidence. 
Table 1. Percentage points of the $A_{200}$ distribution for samples of LG analogues with $-195 \mathrm{~km} \mathrm{~s}^{-1}<V_{\text {ra }}<-65 \mathrm{~km} \mathrm{~s}^{-1}$

\begin{tabular}{lcccccr}
\hline & $5 \%$ & $25 \%$ & $50 \%$ & $75 \%$ & $95 \%$ & \# of pairs \\
\hline $200 \mathrm{~km} \mathrm{~s}^{-1} \leqslant V_{\max }<250 \mathrm{~km} \mathrm{~s}^{-1}$ & & & & & & \\
morphology, isolation & 0.67 & 0.93 & 1.15 & 1.47 & 2.05 & 117 \\
morphology, no isolation & 0.61 & 0.93 & 1.14 & 1.52 & 2.09 & 155 \\
no morphology, isolation & 0.67 & 0.97 & 1.20 & 1.50 & 2.32 & 758 \\
no morphology, no isolation & 0.63 & 0.96 & 1.22 & 1.55 & 2.54 & 1015 \\
\hline $150 \mathrm{~km} \mathrm{~s}^{-1} \leqslant V_{\max }<300 \mathrm{~km} \mathrm{~s}^{-1}$ & & & & & & \\
morphology, isolation & 0.34 & 0.72 & 0.99 & 1.27 & 1.93 & 1273 \\
morphology, no isolation & 0.33 & 0.68 & 0.98 & 1.29 & 2.00 & 1650 \\
no morphology, isolation & 0.41 & 0.81 & 1.09 & 1.40 & 2.21 & 8449 \\
no morphology, no isolation & 0.35 & 0.77 & 1.08 & 1.43 & 2.41 & 11838 \\
\hline
\end{tabular}

Table 2. Percentage points of the $A_{200}$ distribution for samples of LG analogues with $V_{r a}<0$

\begin{tabular}{lcccccr}
\hline & $5 \%$ & $25 \%$ & $50 \%$ & $75 \%$ & $95 \%$ & \# of pairs \\
\hline $200 \mathrm{~km} \mathrm{~s}^{-1} \leqslant V_{\max }<250 \mathrm{~km} \mathrm{~s}^{-1}$ & & & & & & \\
morphology, isolation & 0.54 & 0.97 & 1.33 & 1.66 & 3.93 & 178 \\
morphology, no isolation & 0.45 & 0.94 & 1.26 & 1.66 & 3.93 & 241 \\
no morphology, isolation & 0.54 & 1.01 & 1.34 & 1.82 & 4.62 & 1128 \\
no morphology, no isolation & 0.42 & 0.96 & 1.34 & 1.93 & 5.11 & 1596 \\
\hline $150 \mathrm{~km} \mathrm{~s}^{-1} \leqslant V_{\max }<300 \mathrm{~km} \mathrm{~s}^{-1}$ & & & & & & \\
morphology, isolation & 0.28 & 0.85 & 1.19 & 1.64 & 3.30 & 2815 \\
morphology, no isolation & 0.22 & 0.77 & 1.16 & 1.64 & 3.38 & 3532 \\
no morphology, isolation & 0.31 & 0.89 & 1.23 & 1.76 & 4.06 & 16479 \\
no morphology, no isolation & 0.18 & 0.79 & 1.19 & 1.78 & 4.46 & 23429 \\
\hline
\end{tabular}

\subsection{Application to the Milky Way}

We now calibrate the Zaritsky et al. (1989) Timing Argument which estimates the mass of the Milky Way from the position and velocity of Leo I. This again assumes a radial Keplerian orbit, but Leo I is taken to have passed through pericentre and to be currently moving towards apogalacticon for the second time. Equations (1) to (3) then give a unique mass estimate $M_{M W, T A}$ for the system for any assumed distance and radial velocity. This is taken as the Milky Way's mass since the mass of Leo I is negligible in comparison.

Proceeding as for the Local Group, we select Milky Way Leo I analogues from the Millennium Simulation in order to study the relation of this TA estimate to the true mass of the Milky Way, which we again take to be $M_{200}$. Thus we define the ratio

$B_{200}=M_{200} / M_{M W, T A}$

and investigate its distribution in various samples of analogue hostsatellite systems. In particular, we consider samples of isolated host galaxies (as defined in Section 2) using both our looser and tighter $V_{\max }$ ranges, both with and without cuts on central galaxy morphology, and requiring the distance, radial velocity and maximum circular velocity of the satellite to satisfy $200 \mathrm{kpc}<\mathrm{r}<300 \mathrm{kpc}$, $V_{\text {ra }} \geqslant 0.7 V_{\max }$ (host) and $V_{\max }(\mathrm{comp}) \leqslant 80 \mathrm{~km} \mathrm{~s}^{-1}$.

Results for these four samples are given in Table 3 and the corresponding cumulative distributions of $B_{200}$ are plotted in Fig. 3 Scatter plots of $M_{200}$ against $M_{M W, T A}$ for the four samples are shown in Fig. 4 The behaviour is quite similar to that of the Local Group TA mass estimator $A_{200}$. The median value of $B_{200}$ is robust and varies very little as the definition of the analogue sample is changed. Again it is $10-15 \%$ smaller for samples with the looser $V_{\max }$ selection. Unlike the Local Group case, the median value of $B_{200}$ is about 1.6 and so is significantly larger than unity. This shows that $M_{M W, T A}$ is biased low as an estimator of true Milky Way mass, reflecting the fact that tangential motions are often significant for satellites at the distance of Leo I. Assuming a purely radial orbit then results in an underestimate of the mass.

The width of the distribution of $B_{200}$ is significantly greater for samples with the looser $V_{\max }$ selection, primarily through an extension of the tail towards low values. This resembles the behaviour we saw above for $A_{200}$ but it must have a different cause, since our selection criteria for Milky Way analogues put no upper bound on $V_{r a}$, instead placing a lower limit on $V_{r a} / V_{\max }$. As a result they do not force an upper limit on $M_{M W, T A}$ of the kind imposed on $M_{L G, T A}$ by our upper limit on $V_{r a}$ for Local Group analogues. Fig. 4 shows that the tail of low $B_{200}$ values for the wider $V_{\max }$ range is caused a relatively small number of systems for which $M_{M W, T A}$ is anomalously large. These are objects with anomalously large values of $V_{r a}$ and seem to occur preferentially at small $M_{200}$, corresponding to values of $V_{\max }$ below $200 \mathrm{~km} \mathrm{~s}^{-1}$.

The bulk of the points in Fig. 4 scatter fairly symmetrically about the median relation $M_{200}=1.6 M_{M W, T A}$ which we show as a dashed straight line. Their mean slope is somewhat steeper than strict proportionality because our distance constraint on "Leo I's" is expressed in units of kpc rather than of $R_{200}$ or $V_{\max } / H_{0}$. Distant outliers occur only the low $M_{200}$ side of this relation, suggesting that they may be a consequence of resolution problems in the Millennium Simulation. For $V_{\max } \sim 150 \mathrm{~km} \mathrm{~s}^{-1}$, typical halos are represented by fewer than 1,000 particles and it seems likely that difficulties in describing the dynamics of their satellite substructures may begin to surface. In addition, the sample sizes are relatively small, particularly when we impose a morphology cut, so that 
Table 3. Percentage points of the $B_{200}$ distribution for samples of MW - Leo I analogues with $V_{\text {ra }} \geqslant 0.7 V_{\max }($ host)

\begin{tabular}{lrrrrrr}
\hline & $5 \%$ & $25 \%$ & $50 \%$ & $75 \%$ & $95 \%$ & \# of pairs \\
\hline $200 \mathrm{~km} \mathrm{~s}^{-1} \leqslant V_{\max }($ host $)<250 \mathrm{~km} \mathrm{~s}^{-1}$ & & & & & & \\
$\begin{array}{l}\text { morphology } \\
\text { no morphology }\end{array}$ & 0.71 & 1.27 & 1.71 & 2.01 & 2.62 & 168 \\
\hline $150 \mathrm{~km} \mathrm{~s}^{-1} \leqslant V_{\max }($ host $)<300 \mathrm{~km} \mathrm{~s}^{-1}$ & & 1.25 & 1.67 & 2.01 & 2.55 & 374 \\
$\begin{array}{l}\text { morphology } \\
\text { no morphology }\end{array}$ & 0.39 & 1.04 & 1.50 & 1.89 & 2.47 & 344 \\
\hline
\end{tabular}

the estimates of the tails of the distributions may be noisy. This may explain in part the apparent excess of outliers in the morphologyselected sample with the wider $V_{\max }$ range.

The observational data needed to obtain the TA estimate of the Milky Way's mass are the age of the Universe and the Galactocentric distance and radial velocity of Leo I. As above, we take the age of the Universe to be $13.73 \pm 0.16$ Gyr from Spergel et al. (2007). For the heliocentric distance to Leo I we adopt $254 \pm 19 \mathrm{kpc}$ from Bellazzini et al. (2004). The heliocentric radial velocity of Leo $\mathrm{I}$ is very precisely determined, $283 \pm 0.5 \mathrm{~km} \mathrm{~s}^{-1}$ according to Mateo, Olszewski \& Walker (2007). Based on an assumed Galactic rotation speed at the Sun of $220 \pm 15 \mathrm{~km} \mathrm{~s}^{-1}$, we derive a corresponding Galactocentric radial velocity of $175 \pm 8 \mathrm{~km} \mathrm{~s}^{-1}$. When substituted into Equations (1) to (3), these parameters produce a TA estimate for the Milky Way's mass of

$M_{M W, T A}=1.57 \pm 0.20 \times 10^{12} \mathrm{M}_{\odot}$

As was the case for the Local Group, the fundamental observational quantities are so well defined that the uncertainty of this estimate is much smaller than the expected scatter in $B_{200}$. We will therefore neglect it in the following. The implied apocentric distance of Leo I is $619 \pm 26 \mathrm{kpc}$. Since this is about half the apocentric distance of the M31 - Milky Way relative orbit in the TA model of Section 3.2 perturbations of the orbit of Leo I due to the larger scale dynamics of the Local Group seem quite likely.

For the reasons discussed above, we consider our most precise and robust estimate for the distribution of $B_{200}$ to be that obtained for host galaxies with $150 \mathrm{~km} \mathrm{~s}^{-1} \leqslant V_{\max }$ (host) $<300 \mathrm{~km} \mathrm{~s}^{-1}$ and with no morphology cut. The median of this distribution then gives our best estimate of the true halo mass of the Milky Way:

$M_{200, M W}=2.43 \times 10^{12} \mathrm{M}_{\odot}$,

or $\log M_{200} / M_{\odot}=12.39$. The quartiles of the distribution imply $[12.25,12.49]$ for the most probable range of this quantity, while the $5 \%$ point implies a lower limit of 11.90 at $95 \%$ confidence. Thus the implied mass of the Milky Way is roughly half that of the Local Group as a whole, as might be expected on the basis of the similarity of the two giant galaxies. It is quite similar to other recent estimates based on applying equilibrium dynamics to the system of distant Milky Way satellites and halo stars (e.g.Wilkinson \& Evans 1999; Sakamoto, Chiba \& Beers 2003). A significantly smaller estimate came from the analysis of the high-velocity tail of the local stellar population by Smith et al. (2007), but we note that such analyses, in reality, only place a lower limit on the mass of the halo, since the distribution of solar neighbourhood stars may well be truncated at energies significantly below the escape energy.

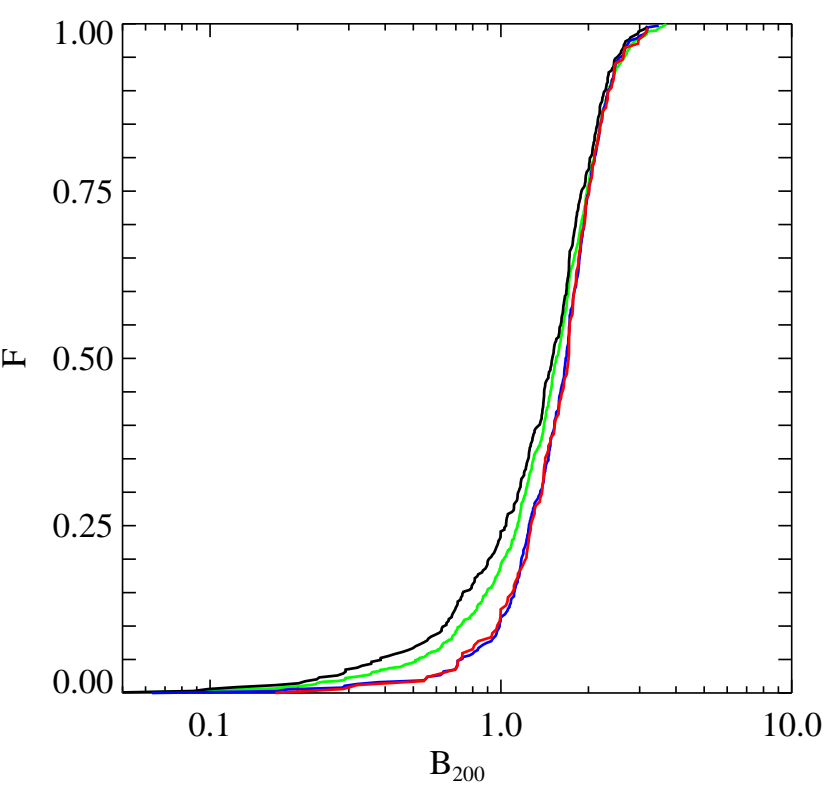

Figure 3. Cumulative distributions of $B_{200}$ the ratio of true Milky Way mass (taken to be $M_{200}$ ) to TA estimate for four samples of isolated Milky Way - Leo I analogues from the Millennium Simulation. The red curve refers to Milky Way analogues with $200 \mathrm{kms}^{-1} \leqslant V_{\max }$ (host) < $250 \mathrm{~km} \mathrm{~s}^{-1}$ and with morphology matching the Milky Way. For the black curve the circular velocity requirement is loosened to $150 \mathrm{~km} \mathrm{~s}^{-1} \leqslant$ $V_{\max }($ host $)<300 \mathrm{~km} \mathrm{~s}^{-1}$, for the blue curve the morphology requirement is removed, and for the green curve both requirements are relaxed. In all cases we require $V_{r a} \geqslant 0.7 V_{\max }$ (host).

\subsection{An alternative mass measure?}

The halo masses we have quoted so far have been based on the "virial masses" $M_{200}$ of simulated halos. This choice is, of course, somewhat arbitrary, and it may not correspond particularly well to the radii within which individual isolated halos are approximately in static equilibrium. As an alternative convention, we here consider defining the mass of an individual halo to be that of the corresponding self-bound subhalo identified by the SUBFIND algorithm of Springel et al. (2001). This algorithm typically includes material outside the radius $R_{200}$ within which $M_{200}$ is measured, but it excludes any material which is identified as part of a smaller subhalo orbiting within the larger system. In this paper we denote this subhalo mass as $M_{\text {halo }}$.

In the left panel of Fig. 5 we plot $M_{\text {halo }}$ against $M_{200}$ for all halos in our preferred sample of Local Group analogues, that with our preferred morphology, isolation and radial velocity cuts and 

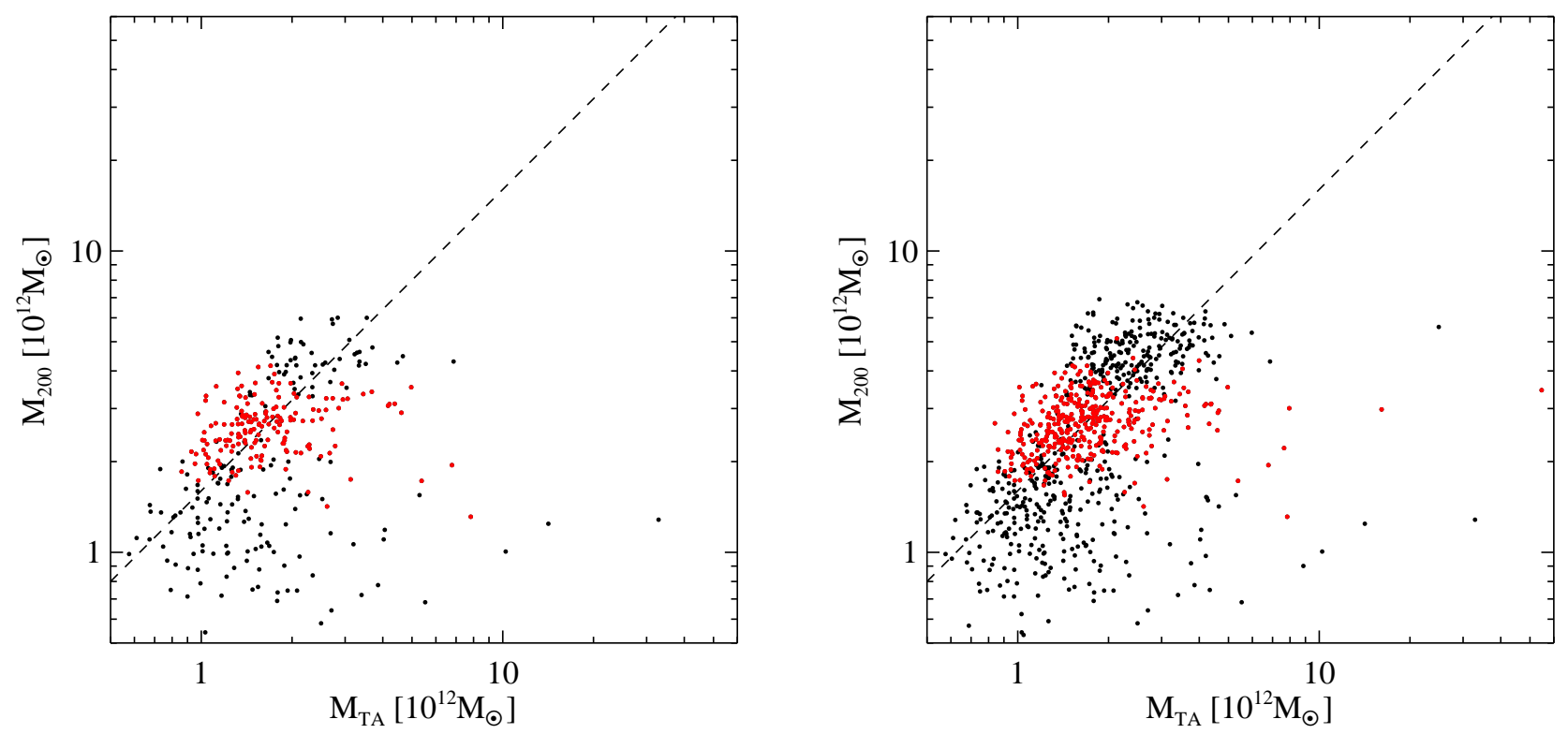

Figure 4. Scatter plots of the TA estimate of the "Milky Way's" mass against its true mass, $M_{200}$. The left panel is for isolated host galaxies with morphology matching that of the real Milky Way, while the right panel is for samples with no such morphology restriction. In both panels red dots refer to simulated host galaxies with $200 \mathrm{~km} \mathrm{~s}^{-1} \leqslant V_{\max }$ (host) $<250 \mathrm{~km} \mathrm{~s}^{-1}$, while black dots indicate other hosts in the broader range $150 \mathrm{~km} \mathrm{~s}{ }^{-1} \leqslant V_{\text {max }}($ host $)<$ $300 \mathrm{~km} \mathrm{~s}^{-1}$. In all cases the analogue of Leo I is required to have $200 \mathrm{kpc}<\mathrm{r}<300 \mathrm{kpc}, V_{\operatorname{ra}} \geqslant 0.7 V_{\max }($ host$)$ and $V_{\max }(\mathrm{comp}) \leqslant 80 \mathrm{~km} \mathrm{~s}-1$. The straight dashed lines in the two panels show the approximate median relation $M_{200}=1.6 M_{M W, T A}$

with $150 \mathrm{~km} \mathrm{~s}^{-1} \leqslant V_{\max }<300 \mathrm{~km} \mathrm{~s}^{-1}$. Black and red points in this plot refer to Type 0 and Type 1 subhalos respectively. The right panel of Fig. [5 is a similar plot for the "Milky Way" halos in our preferred sample of Milky Way - Leo I analogues, again the sample which is matched in morphology and which has the wider $V_{\max }$ range. In both panels it is clear that the correspondence between the two mass definitions is quite tight, and that $M_{\text {halo }}$ tends to be somewhat larger than $M_{200}$. In addition the left panel shows that Type 1 halos have smaller $M_{\text {halo }}$ for given $M_{200}$ than do Type 0 halos, as would naively be expected. The average value of $\log M_{\text {halo }} / M_{200}$ for the halos in the left panel is 0.100 for the Type 0's and -0.004 for the Type 1's, while it is 0.079 for the "Milky Way" halos in the right panel.

This close correspondence between the two mass definitions carries over to the distribution of our ratios of "true" mass to timing mass. In Table 4 we give percentage points of the $A_{\text {halo }}$ distribution for the 8 samples of Local Group analogues already considered above. They can be compared directly with the numbers given in Table 1 for these samples. To a good approximation the distribution of $A_{\text {halo }}$ agrees with that of $A_{200}$ except that all values are shifted upwards by about $16-20 \%$.

The same is also true for estimates of the Milky Way's mass obtained using the TA applied to Leo I. This can be seen from Table 5 which repeats Table 3 except that we now give percentage points for $B_{\text {halo }}$ rather than $B_{200}$. Clearly it is of rather little importance which definition of halo mass we adopt: the results obtained with our two definitions are very similar.

\section{DISCUSSION AND CONCLUSIONS}

The statistical argument underlying the analysis of this paper is more subtle than it may at first appear, so it is worth restating it somewhat more formally in order to understand what is being assumed in deriving the mass estimates for the Local Group and for the Milky Way given above.

We believe that the mass distributions around galaxies are much more extended than the visible stellar distributions, and that these have been assembled from near-uniform "initial" conditions in a manner at least qualitatively resembling that in a $\Lambda C D M$ universe. Thus the assembly histories of the Local Group and of the Milky Way's halo differ in major ways from those assumed by the original Timing Arguments of Kahn \& Woltier (1959) and Zaritsky et al. (1989). In addition, the meaning of the derived mass values needs clarification. We wish to use the Millennium Simulation to calibrate the TA estimates against conventional measures of halo mass, and to test the general applicability of the Timing Argument. However, we want to do this in a way which avoids any significant dependence on the details of the $\Lambda$ CDM model, for example, on the exact density profiles, abundances and substructure properties which it predicts for halos.

Our method uses the simulation to estimate the distribution of the ratio of "true" mass to TA mass estimate for samples of objects whose properties "resemble" those of the observed Local Group and Milky Way - Leo I systems. Our restrictions on separation and radial velocity implement this similarity requirement in a straightforward way, but our constraints on $V_{\max }$ have a more complex effect. Although the true $V_{\max }$ values for M31 and the Milky Way are very likely within our looser range $\left(150 \mathrm{~km} \mathrm{~s}^{-1} \leqslant\right.$ $V_{\max }<300 \mathrm{~km} \mathrm{~s}^{-1}$ ) the simulation exhibits a tight correlation between $V_{\max }$ and $M_{200}$. Imposing fixed limits on $V_{\max }$ is thus effectively equivalent to choosing a fixed range of $M_{200}$. As a result, we are in practice estimating the distribution of $A_{200}$ or $B_{200}$ for systems of given true mass, subject to the assumed constraints on separation and radial velocity. However, when we apply our results to estimate true masses for the Local Group and the Milky 

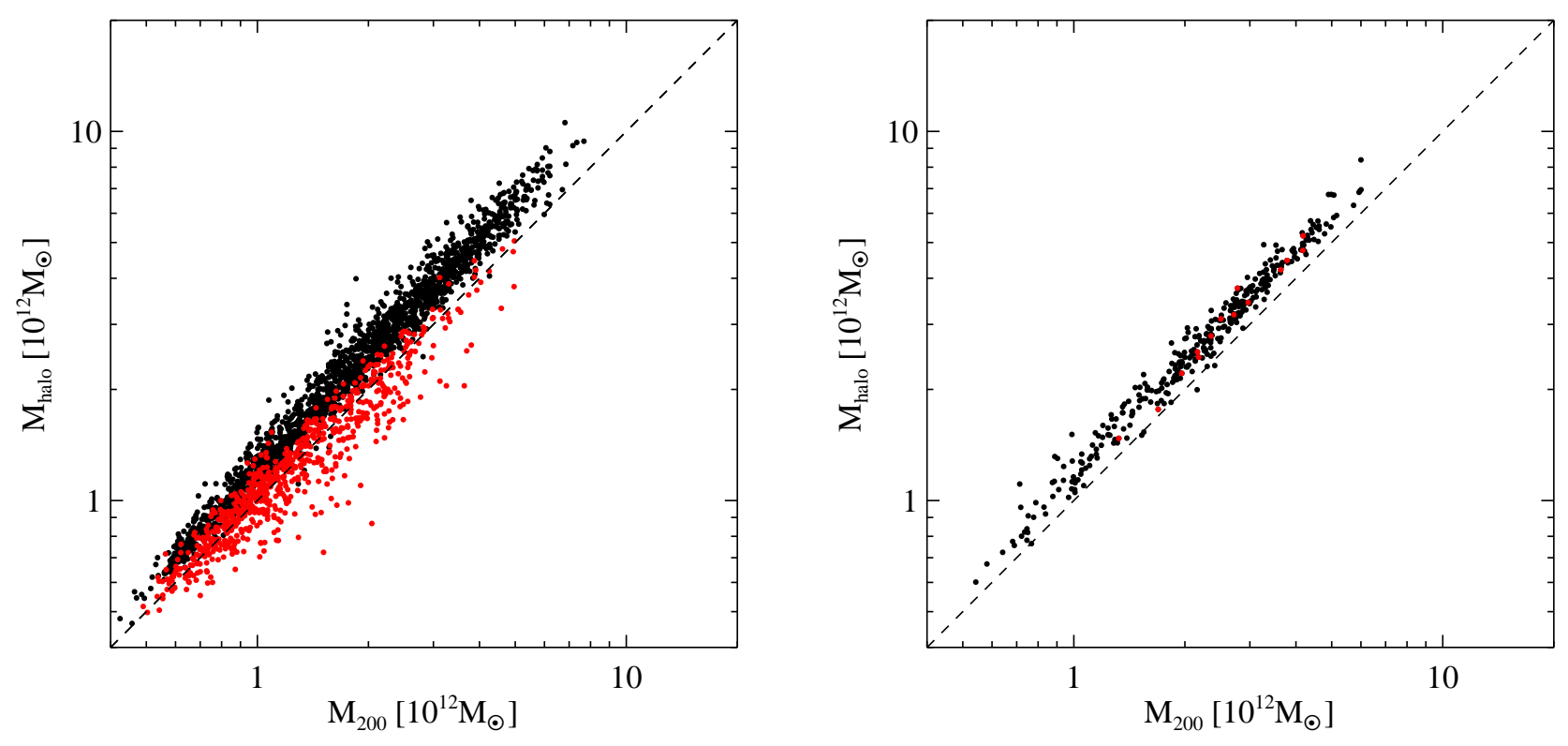

Figure 5. In the left panel we plot $M_{\text {halo }}$ against $M_{200}$ for all the galaxy (sub)halos in our sample of Local Group analogues with our preferred morphology, isolation and radial velocity cuts and with $150 \mathrm{~km} \mathrm{~s}^{-1} \leqslant V_{\max }<300 \mathrm{~km} \mathrm{~s}^{-1}$. Type 0 subhalos are plotted black while Type 1 subhalos are red. The diagonal line is the one-to-one relation. The right panel is a similar plot in for the "Milky Way" halos in our preferred sample of Milky Way - Leo I analogues.

Table 4. Percentage points of the $A_{\text {halo }}$ distribution for samples of LG analogues with $-195 \mathrm{~km} \mathrm{~s}^{-1}<V_{\text {ra }}<-65 \mathrm{~km} \mathrm{~s}^{-1}$

\begin{tabular}{lcccccr}
\hline & $5 \%$ & $25 \%$ & $50 \%$ & $75 \%$ & $95 \%$ & \# of pairs \\
\hline $200 \mathrm{~km} \mathrm{~s}^{-1} \leqslant V_{\max }<250 \mathrm{~km} \mathrm{~s}^{-1}$ & & & & & & \\
morphology, isolation & 0.78 & 1.11 & 1.36 & 1.72 & 2.38 & 117 \\
morphology, no isolation & 0.69 & 1.14 & 1.36 & 1.77 & 2.39 & 155 \\
no morphology, isolation & 0.76 & 1.17 & 1.44 & 1.75 & 2.74 & 758 \\
no morphology, no isolation & 0.71 & 1.15 & 1.44 & 1.82 & 2.97 & 1015 \\
\hline $150 \mathrm{~km} \mathrm{~s}^{-1} \leqslant V_{\max }<300 \mathrm{~km} \mathrm{~s}^{-1}$ & & & & & & \\
morphology, isolation & 0.41 & 0.88 & 1.20 & 1.53 & 2.26 & 1273 \\
morphology, no isolation & 0.38 & 0.84 & 1.19 & 1.54 & 2.38 & 1650 \\
no morphology, isolation & 0.49 & 0.99 & 1.31 & 1.67 & 2.60 & 8449 \\
no morphology, no isolation & 0.37 & 0.92 & 1.30 & 1.71 & 2.85 & 11838 \\
\hline
\end{tabular}

Way, we implicitly assume that our distributions of $A_{200}$ and $B_{200}$ are appropriate for samples of given TA mass estimate, again subject to our constraints on separation and radial velocity. It is thus important to understand when these two distributions can be considered the same.

The relation can be clarified as follows. From the simulation we compile the distribution of $M_{t r} / M_{T A}$, or equivalently of $\Delta \equiv \ln M_{t r}-\ln M_{T A}$, for systems with $\ln M_{t r}$ in a given range. We then implicitly assume that this distribution does not depend on $M_{t r}$, at least over this range, so that the result can be taken as an estimate of the probability density of $\Delta$ at given $M_{t r}$. Bayes Theorem then gives us the probability density function (pdf) for $\Delta$ at fixed $M_{T A}$ :

$$
\begin{aligned}
f\left[\Delta \mid \ln M_{T A}\right] & =\frac{f\left[\Delta, \ln M_{T A}\right]}{f\left[\ln M_{T A}\right]} \\
& =\frac{f\left[\Delta, \ln M_{t r}\right]}{f\left[\ln M_{T A}\right]}
\end{aligned}
$$

$$
\begin{array}{r}
=\frac{f\left[\Delta \mid \ln M_{t r}\right] f\left[\ln M_{t r}\right]}{f\left[\ln M_{T A}\right]} \\
=f\left[\Delta \mid \ln M_{t r}\right]
\end{array}
$$

The first line here simply writes the conditional pdf of $\Delta$ at given $M_{T A}$ in terms of the joint pdf of the two quantities and the pdf of $M_{T A}$. The second line then rewrites the joint pdf in terms of the equivalent variables $\Delta$ and $M_{t r}$, using the fact that the Jacobian of the transformation is unity. The third line re-expresses the joint pdf as the product of the pdf of $\Delta$ at given $M_{t r}$ times the pdf of $M_{t r}$. The final line then follows from the normalisation condition, provided that $f\left[\ln M_{t r}\right]$ is constant and $f\left[\Delta \mid \ln M_{t r}\right]$ is independent of $M_{t r}$. Thus, when estimating $M_{t r}$ from $M_{T A}$, we assume a uniform prior on $\ln M_{t r}$ and that the distribution of $\Delta$ does not depend on true mass. Both these assumptions appear natural and appropriate.

The analysis underlying the Timing Argument (Equations (1) - (3)) assumes that the relative orbit of the two objects is bound and has conserved energy since the Big Bang. Recently, Sales et al. (2007) have shown that in $\Lambda$ CDM models this assumption is signif- 
Table 5. Percentage points of the $B_{\text {halo }}$ distribution for samples of MW - Leo I analogues with $V_{\text {ra }} \geqslant 0.7 V_{\max , M W}$

\begin{tabular}{lcrrrrr}
\hline & $5 \%$ & $25 \%$ & $50 \%$ & $75 \%$ & $95 \%$ & \# of pairs \\
\hline $200 \mathrm{~km} \mathrm{~s}^{-1} \leqslant V_{\max }<250 \mathrm{~km} \mathrm{~s}^{-1}$ & & & & & & \\
$\begin{array}{l}\text { morphology } \\
\text { no morphology }\end{array}$ & 0.90 & 1.53 & 1.98 & 2.40 & 3.14 & 168 \\
\hline $150 \mathrm{~km} \mathrm{~s}^{-1} \leqslant V_{\max }<300 \mathrm{~km} \mathrm{~s}^{-1}$ & & 1.50 & 1.97 & 2.39 & 3.21 & 374 \\
morphology & 0.42 & 1.25 & 1.81 & 2.31 & 3.09 & 344 \\
no morphology & 0.61 & 1.37 & 1.88 & 2.36 & 3.23 & 896 \\
\hline
\end{tabular}

icantly violated for a substantial number of satellites within halos comparable to that of the Milky Way. In particular, they demonstrate the presence of a tail of unbound objects which are being ejected from halos as a result of 3-body "slingshot" effects during their first pericentric passage. These objects are typically receding rapidly from their "Milky Way", as assumed in the Zaritsky et al. (1989) argument, but they violate its assumption that the present orbital energy can be used to infer the period of the initial orbit (i.e. the time from the Big Bang to first pericentric passage). Clearly such objects should also be present in the Millennium Simulation, although lack of resolution might make them under-represented in comparison to the simulations analysed by Sales et al. (2007). Thus our analysis takes the possibility of such ejected satellites into account, at least in principle. Objects of this type will show up as systems with anomalously large TA estimates for their halo mass, and Fig. 4. shows a number of outliers which could well be explained in this way. Issues of this kind do not effect TA-based estimates of the mass of the Local Group since the two big galaxies are currently approaching for the first time.

The only kinematic information about the relative orbit of M31 and the Milky Way used in our analysis is their current approach velocity. van der Marel \& Guhathakurta (2007) show that geometric arguments can already constrain the transverse component also, and future astrometry missions such as SIM might allow $V_{t r}$ to be measured directly. Thus it is interesting to ask if our TA mass estimate could be significantly refined by measuring the full 3-D relative motion of the two galaxies, rather than just its radial component. We address this in Fig. 6 which plots $A_{200}$, the ratio of true mass to TA estimate, against $V_{t r}$ for a sample of Local Group analogues with our preferred morphology, isolation and radial velocity cuts, and with $150 \mathrm{~km} \mathrm{~s}^{-1} \leqslant V_{\max }<300 \mathrm{~km} \mathrm{~s}^{-1}$. The median $V_{t r}$ for this sample is $86 \mathrm{~km} \mathrm{~s}^{-1}$, comparable to the van der Marel \& Guhathakurta (2007) estimate for the real system. There is no apparent correlation of $A_{200}$ with $V_{t r}$, and indeed, the medians of $A_{200}$ for the high and low $V_{t r}$ halves of the sample are both close to 1 and do not differ significantly. Pairs with high $V_{t r}$ do show larger scatter in $A_{200}$ than pairs on near-radial orbits. For given separation, radial velocity and age, the Kepler model implies a mass which increases monotonically with $V_{t r}$. The absence of a detectable trend in Fig. 6 shows that uncertainties in $V_{t r}$ do not dominate the scatter in our TA mass estimate, and that a measurement of $V_{t r}$ will not substantially increase the precision with which the true mass can be measured.

In conclusion, our analysis shows the Timing Argument to produce robust estimates of true mass both for the Local Group and for the Milky Way, as long as "true mass" is understood to mean the sum of the conventional masses of the major halos. For the Local Group as a whole, the estimate and confidence limits given in Section 3.2 and in the Abstract appear reliable given the excel-

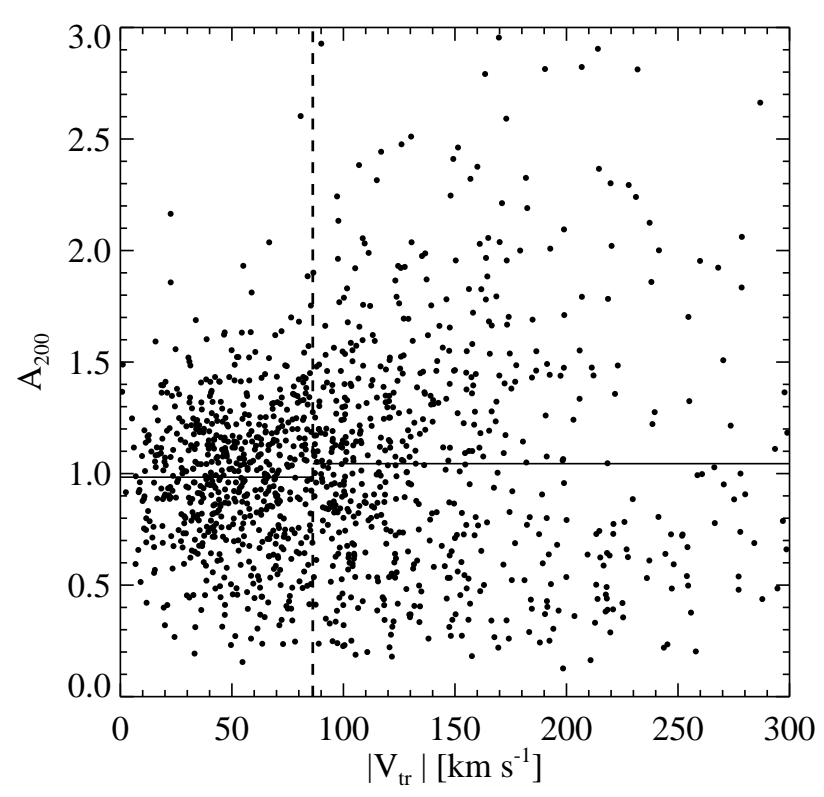

Figure 6. Scatter plot of $A_{200}$ versus transverse velocity for Local Group analogues in our sample with preferred morphology, isolation and radial velocity cuts and with $150 \mathrm{~km} \mathrm{~s}^{-1} \leqslant V_{\max }<300 \mathrm{~km} \mathrm{~s}^{-1}$. The vertical dashed line indicates the median $V_{t r}$. The distributions on either side of this line are each further split in half at the median values of $V_{t r}$ (the solid horizontal lines.) There is essentially no correlation in this plot, indicating that a measurement of the transverse velocity will not significantly improve the TA mass estimate.

lent statistics provided by the Millennium Simulation, the lack of any substantial dependence on our isolation and morphology cuts, and the relatively simple dynamical situation. Although the results based on Leo I's orbit also appear statistically sound, the more complex dynamical situation offers greater scope for uncertainty, particularly when trying to place a lower limit on the mass of the Milky Way's halo. On the other hand, our best estimate of this mass is just under half of our estimate of the sum of the halo masses of M31 and the Galaxy. Thus the picture presented by the data appears quite consistent, and gives no reason to be suspicious of the Milky Way results.

\section{ACKNOWLEDGEMENTS}

We thank Andreas Faltenbacher for help in calculating the $M_{200}$ values of Type 1 halos; the referee, John Dubinski, for useful suggestions regarding the transverse velocity. YSL also thanks Martin 
C. Smith, Gabriella De Lucia and Amina Helmi for useful discussions, and the Max Planck Institute for Astrophysics for support during her visits there. SW thanks the Kapteyn Astronomical In-

stitute for support as Blaauw Lecturer during extended visits to Groningen.

\section{REFERENCES}

Bellazzini M., Gennari N., Ferraro F.R., Sollima A., 2004, MNRAS, 354, 708

Blumenthal G.R., Faber S.M. Primack J.R.,Rees M.J., 1984, Nature, 311, 517

Bower R.G. et al., 2006, MNRAS, 370, 645

Courteau S., van den Bergh S., 1999, AJ, 118, 337

Croton D.J. et al., 2006, MNRAS, 365, 11

Davis M., Efstathiou G., Frenk C.S., White S.D.M., 1985, ApJ, 292, 371

Einasto J., Kaasik A., Saar E., 1974, Nature, 250, 309

Einasto J., Lynden-Bell D., 1982, MNRAS, 199, 67

Holland S., 1998, AJ, 115, 1916

Kahn F.D., Woltjer L., 1959, ApJ, 130, 705

Karachentsev I.D., Kashibadze O.G., 2005, astro-ph/0509207

Kauffmann G., Colberg J.M., Diaferio A., White S.D.M., 1999, MNRAS, 303, 188

Kitzbichler M.G., White S.D.M., 2006, MNRAS, 366, 858

Knapp G.R., Stark A.A., Wilson R.W., 1985, AJ, 90, 254

Kochanek C.S., 1996, ApJ, 457, 228

Kroeker T.L., Carlberg G., 1991, ApJ, 376, 1

De Lucia G., Blaizot J., 2007, MNRAS, 375, 2

Mandelbaum R. et al., 2006, MNRAS, 368, 715

Mateo M., Olszewski E.W., Walker M.G., 2007, ApJ accepted (astro-ph/0708.1327)

Navarro J.F., Frenk C.S., White S.D.M., 1997, ApJ, 490, 493

Ostriker J.P., Peebles P.J.E., Yahil A., 1974, ApJ, 193, 1

Lynden-Bell D., 1981, Obs, 101, 111

Perlmutter S. et al., 1999, ApJ, 517, 565

Prada F. et al., 2003, ApJ, 598, 260

Riess A.G. et al., 1998, AJ, 116, 1009

Sakamoto T., Chiba M., Beers T.C., 2003, A\&A, 397, 899

Sales L.V., Navarro J.F., Abadi M.G. Steinmetz M., 2007, MNRAS, 379, 1475

Seljak U., 2002, MNRAS, 334, 797

Smith M.C. et al, 2007, MNRAS, 379, 755

Smoot G.F. et al, 1992, ApJ, 396, 1

Spergel D.N. et al., 2003, ApJS, 148, 175

Spergel D.N. et al., 2007, ApJS, 170, 377

Springel V. et al., 2005, Nature, 435, 629

Springel V., White S.D.M., Tormen G., Kauffmann G., 2001, MNRAS, 328, 726

Stanek K.Z., Garnavich P.M., 1998, ApJ, 503L, 131

van der Marel R.P., Guhathakurta P., 2007, ApJ submitted (astro$\mathrm{ph} / 0709.3747)$

Wilkinson M.I., Evans N.W., 1999, MNRAS, 310, 645

White S.D.M., Rees M.J., 1978, MNRAS, 183, 341

Zaritsky D. et al., 1989, ApJ, 345, 759

Zaritsky D., Smith R., Frenk C., White S.D.M., 1997, ApJ, 478, 39 